\title{
Photolithographically Home-Made PVDF Sensor for Cavitation Impact Load Measurement
}

\author{
Jan Hujer ${ }^{1, *}$, Menghuot Phan $^{1}$, Tomáš Kořínek ${ }^{1}$, Petra Dančová ${ }^{1}$, and Miloš Müller ${ }^{1}$ \\ ${ }^{1}$ Technical University of Liberec, Faculty of Mechanical Engineering, Department of Power \\ Engineering Equipment, Studentská 2, 46117 Liberec, Czech Republic
}

\begin{abstract}
Piezoelectric PVDF sensors offer a unique option for the measurement of cavitation aggressiveness represented by the magnitude of impacts due to cavitation bubble collapses near walls. The aggressiveness measurement requires specific sensors shape and area, whereas commercial PVDF sensors are fabricated in limited geometry and size ranges. The photolithography method offers a possibility of production of homemade PVDF sensors of arbitrary shape and size. This paper deals with the calibration of a photolithographically home-made PVDF sensor for the cavitation impact load measurement. The calibration of sensors was carried out by the ball drop method. Sensors of different sizes were fabricated by the photolithography method from multi-purpose both side metallized PVDF sheet. The standard technology used for the fabrication of printed circuit boards was utilized. Commercial PVDF sensors of the same size were calibrated and the calibration results were compared with the home-made sensors. The effect of size and the effect of one added protective layer of Kapton tape on a sensor sensitivity were investigated.
\end{abstract}

\section{Introduction}

The strong noise, vibration, loss in the efficiency and the material erosion are effects associated with cavitation phenomenon in the field of turbomachinery. These undesirable effects are caused by collapsing cavitation bubbles or cavitation bubble clouds close to the material surfaces. During the final stage of the collapse, microjets and shock waves are formed. These effects accompanying the bubble collapse impact and erode the surfaces causing the material damage and mass loss. The study of impacts of microjets and shock waves is done on both material and fluid side. On material side the pitting tests are usually performed. Pitting tests are based on the study of micro deformations and their geometries formed during the impact loads. These tests are connected with properties of the tested material. On fluid side it is possible to measure directly cavitation impact loads by suitable sensors [1]. The time scale of these impacts is in order of microsecond [2], thus a high resonant frequency in the order of $\mathrm{MHz}$ and a resistance during the cavitation impact load are required from the sensors.

Several researchers measured cavitation impact forces by piezoelectric sensors. The high resonance frequency of these sensors is well suited to the short time duration of the cavitation

\footnotetext{
* Corresponding author: jan.hujer@tul.cz
} 
phenomenon. Franc et al. [3] measured impact loads in a high-speed cavitation tunnel with a compact commercial pressure sensor (PCB 108A02) of resonant frequency about $250 \mathrm{kHz}$. Authors showed that the used sensor was not optimal because of the limited resonance frequency. Other researchers used piezoelectric polyvinylidene fluorine (PVDF) film sensors. Momma et al. [4] developed a $110 \mu \mathrm{m}$ thick PVDF film sensor which was mounted on a stainless-steel support. The sensor was protected on top by $70 \mu \mathrm{m}$ thick polyamide tapes from water and cavitation damage in the cavitating jet apparatus. These protective layers can however influence the sensor sensitivity. Authors tested effect of two, four and six protective layers of polyimide tapes on pulse height distribution, however the output in all cases was indistinguishable. Soyama et al. [5] used a $110 \mu \mathrm{m}$ thick PVDF film sensor to measure pulses in cavitating jet apparatus. The sensor was protected by $60 \mu \mathrm{m}$ thick polyimide tape. Wang et al. [6] used $28 \mu \mathrm{m}$ thick PVDF film transducer to measure impact forces due to single bubble collapses. Authors protected sensor by a one layer of scotch tape which resulted to sensitivity $0.013 \mathrm{~V} / \mathrm{N}$. Grinspan et al. [7] used $52 \mu \mathrm{m}$ thick PVDF film transducer protected by the cellophane tape. The sensor sensitivity was $0.116 \mathrm{~V} / \mathrm{N}$.

The utilization of the commercial sensors sets the limits in parameters and also for the installation. A promising opportunity is to fabricate a PVDF sensor photolithographically from a sheet of a PVDF film which implies optionally defined size of a sensor or even a matrix of sensors. The aim of this study is to produce, test and calibrate a home-made PVDF sensor for cavitation impact load measurement and compare it with the commercial PVDF sensor (DT1-028K/L, manufactured by TE Connectivity) of the same size used by Wang et al. [6] and Hujer et al. [8]. The influence of a protective layer thickness on the home-made PVDF sensor sensitivity is also evaluated.

\section{Fabrication of PVDF sensors}

The sketch of a home-made sensor composition with basic dimensions is illustrated in the Figure 1. The sensor was made from a multi-purpose both side metallized piezo PVDF sheet (TE Connectivity, part number 1-1003702-7). The piezo film thickness was $28 \mu \mathrm{m}$ with $800 \AA \AA$ of $\mathrm{Cu}-\mathrm{Ni}$ metallization, consisting of a $700 \AA \AA$ copper layer and a $100 \AA$ nickel layer. The standard photolithography process was used for fabrication. This method is used in the production of printed circuit boards. The sensor production workflow included several steps. Firstly, a photosensitive film was joint to metallization of the PVDF film on both side by Laminator RLM 419P. In the next step a photomask in CAD software was created and then printed on a glass plate. The photomask was used to define the shape and size of the sensor. The PVDF sheet with the photosensitive film was then put between two photomasks and UV light was applied for 80 seconds in the exposure unit Bungard. The development of photoresist was done with sodium carbonate solution $\left(\mathrm{Na}_{2} \mathrm{CO}_{3}\right)$ by spraying for 150 seconds. The next steps included the etching in ferric chloride solution $\left(\mathrm{FeCl}_{3}\right)$ for 10 second and removing the remaining photoresist with sodium hydroxide $(\mathrm{NaOH})$. Finally, copper wires for both top and bottom electrodes were riveted.

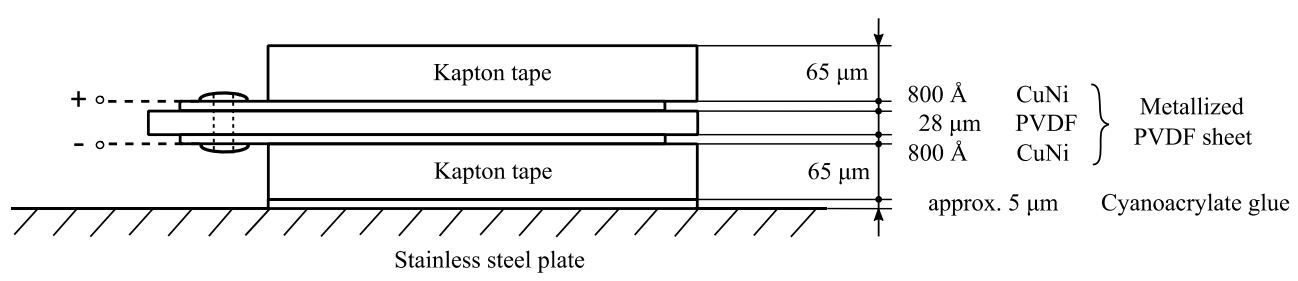

Fig. 1. The home-made sensor composition. 
Before the sensor mounting, uncovered sensor electrodes were electrically insulated by a $65 \mu \mathrm{m}$ thick Kapton tape (KPT 201-2540). Sensors were glued on stainless steel plate by cyanoacrylate glue (Loctite Super Attak Power Flex Gel). The thickness of the glue layer is approximately $5 \mu \mathrm{m}$.

\section{Testing method of PVDF sensors}

The testing of home-made PVDF sensors was conducted by ball drop method which is commonly used for the calibration of impact transducers [9]. The testing method is illustrated in the Figure 2. A stainless-steel ball of a certain weight $m$ drops down vertically from initial position 1 , hits the calibrated sensor and rebounds to position 2. Initial and final ball positions $h_{1}$ and $h_{2}$ are read on scale of rule recorded by camera during each test. A glass tube is used for the ball guiding. During the ball impact, a part of the initial ball energy is transformed in a deformation of sensor. The sensor generates an electric charge which is directly measured as an electric voltage output. The maximum voltage $U_{\max }$ and the time duration of the impact $\tau$ are determined from measured signal.

The calculation of mean impact force $F_{\text {mean }}$ is based on the impulse-momentum theorem expressed by the following formula

$$
F_{\text {mean }}=\frac{1}{\tau} \int_{t_{1}}^{t_{2}} F(t) d t=\frac{m}{\tau}\left(v_{1}+v_{2}\right)
$$

Velocities before and after the ball impact are calculated from the formula

$$
v_{1,2}=\sqrt{2 g h_{1,2}}
$$

where $h_{1,2}$ are initial and final heights of the ball and $g$ is gravity acceleration. Maximum force $F_{\max }$ results from the shape of the measured signal and is considered as double of the mean force $F_{\text {mean }}$ as

$$
F_{\text {max }}=2 \cdot F_{\text {mean }}
$$

\section{Experimental setup}

Two types of sensors were tested in this study. The home-made PVDF sensor described above in details and a commercial PVDF film transducer (DT1-028K/L) manufactured by TE Connectivity. The active area of the commercial sensor is $12 \mathrm{~mm}$ x $30 \mathrm{~mm}$. For the comparison the home-made PVDF sensor with active area $12 \mathrm{~mm}$ x $30 \mathrm{~mm}$ was fabricated. To evaluate the influence of the active sensor area two sensors $12 \mathrm{~mm} \times 10 \mathrm{~mm}$ and $12 \mathrm{~mm} \times 1 \mathrm{~mm}$ were fabricated. The commercial sensor consists of $28 \mu \mathrm{m}$ thick PVDF film with silver ink electrodes and protective urethane coating over electrodes. The total thickness of the sensor was $40 \mu \mathrm{m}$.

The sketch of the experimental setup is in the Figure 2. The tested sensors were connected directly to the input of the acquisition card of the 12- bit NI USB 5105 digitizer controlled by a computer. Acquired signals were analyzed in the software LabVIEW Signal Express. The impact duration $\tau$ and maximum voltage $U_{\max }$ were detected. All devices, the digitizer, computer, stainless steel plate and negative pole of PVDF transducer were grounded to avoid a signal noise. The impact duration $\tau$ was evaluated as the full width at the half of the signal maximum. The ball drop testing of sensors was conducted with four sizes of stainless-steel ball $(0,132 ; 0,260 ; 0,441 \mathrm{~g} ; 0,700 \mathrm{~g})$ and with three glass tubes. 


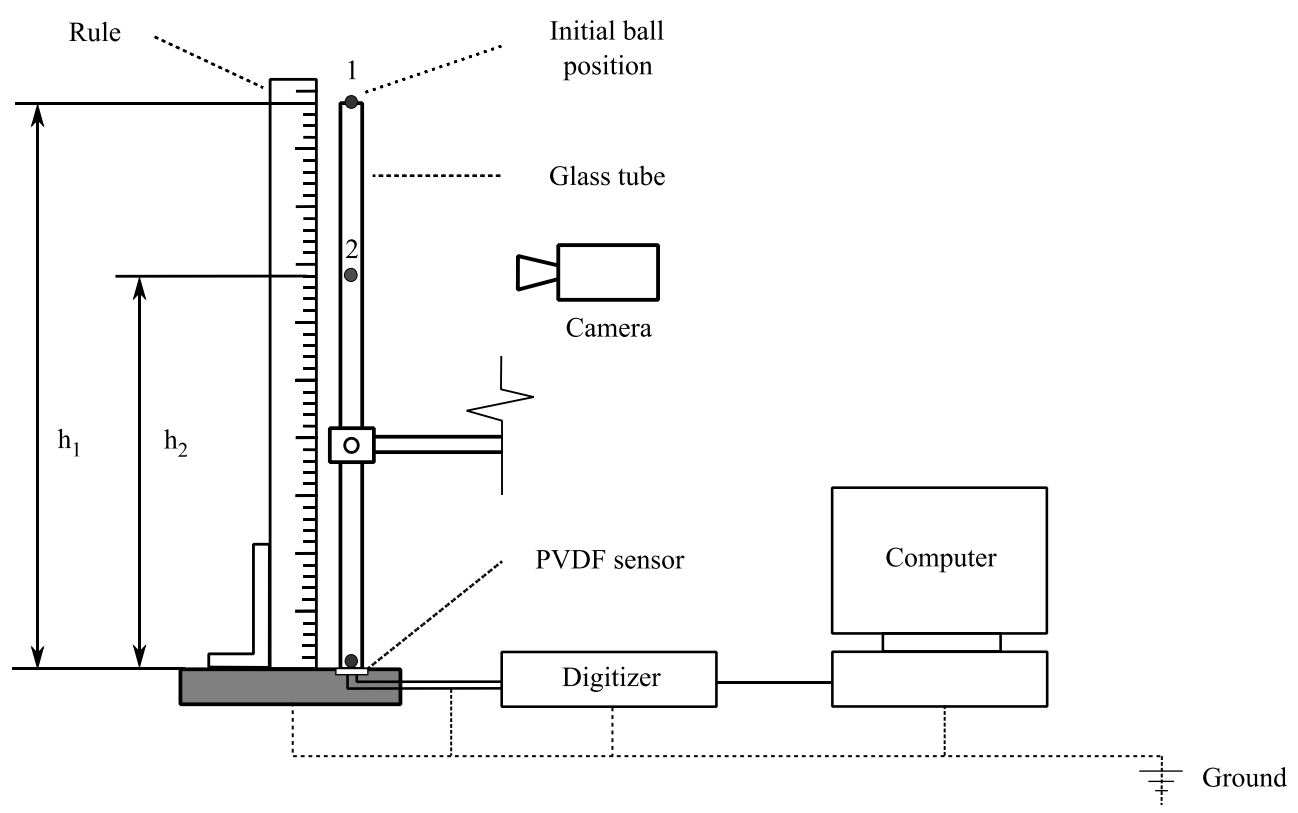

Fig. 2. The ball drop testing method - experimental setup.

The glass tubes length determines the initial ball positions $h_{1}(150 \mathrm{~mm} ; 300 \mathrm{~mm}$; $400 \mathrm{~mm}$ ). The final ball position after the rebound was recorded by the camera. The digitizer sampling frequency was set $10 \mathrm{MHz}$ and input impedance was set $10 \mathrm{M} \Omega$.

\section{Results}

The dependence of the measured maximum voltage $U_{\max }$ and calculated mean impact force $F_{\text {mean }}$ followed a linear trend over all measurements. Therefore, the linear least square method was used to fit the data. Figure 3 shows the determination of the sensitivity constant on the measured data for home-made PVDF sensor of size $12 \mathrm{~mm}$ x $30 \mathrm{~mm}$.

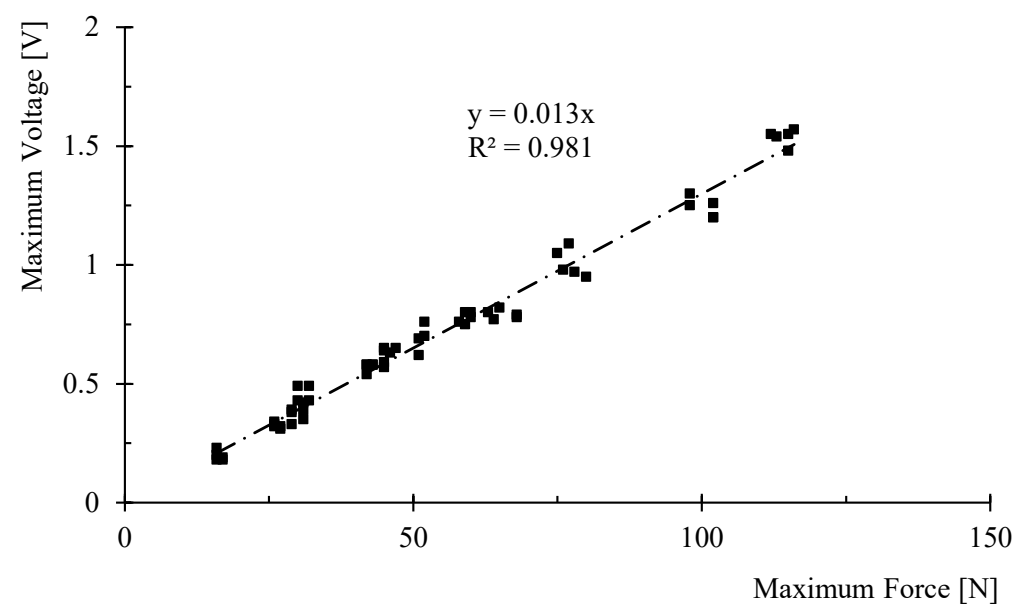

Fig. 3. The dependence of the measured maximum voltage $U_{\max }$ on calculated mean impact force $F_{\text {mean }}$ for the home-made PVDF sensor (12 $\mathrm{mm}$ x $30 \mathrm{~mm}$ ). 


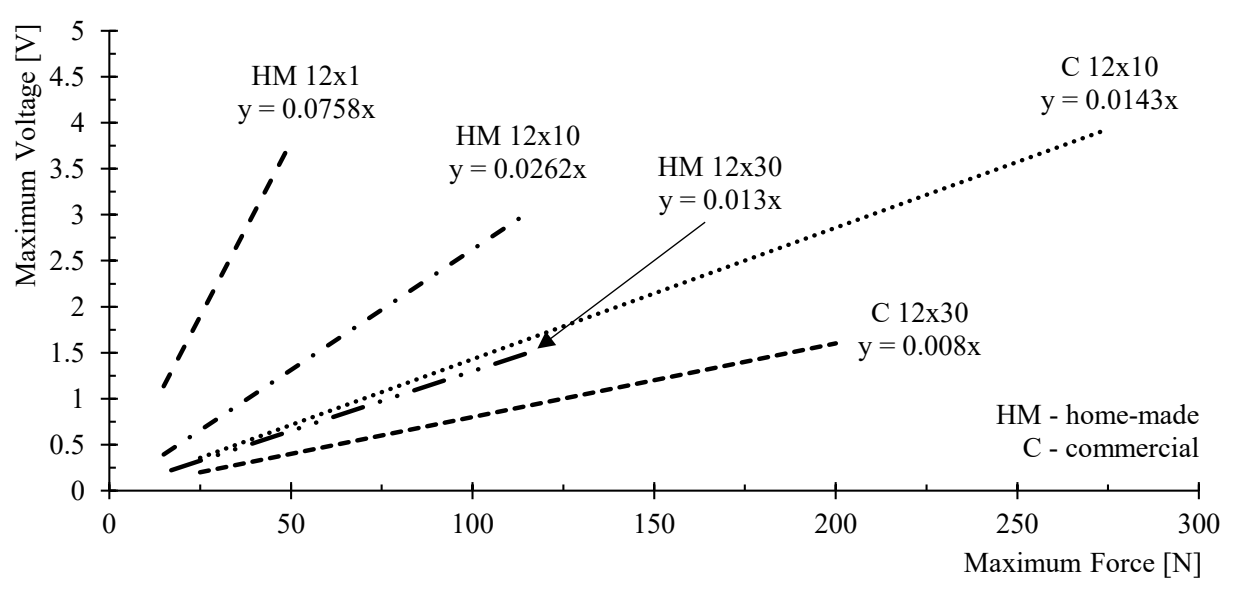

Fig. 4. The comparison of the calibration lines and sensitivities for the home-made PVDF sensors and commercial PVDF sensors for different sensors active areas.

The linear fit gives sensitivity $0.013 \mathrm{~V} / \mathrm{N}$ with the correlation coefficient 0.981 . For the sensitivity constant fitting the condition of the zero maximum voltage at zero maximum force is considered.

The comparison of the calibration lines and sensitivities for the home-made PVDF sensors and commercial PVDF sensors for different sensors active areas is plotted in Figure 4. The sensitivities of home-made PVDF sensors of size $12 \mathrm{~mm} \times 30 \mathrm{~mm}$; $12 \mathrm{~mm} \times 10 \mathrm{~mm}$ and $12 \mathrm{~mm} \times 1 \mathrm{~mm}$ were $0.013 \mathrm{~V} / \mathrm{N} ; 0.0262 \mathrm{~V} / \mathrm{N}$ and $0.0758 \mathrm{~V} / \mathrm{N}$ respectively. The sensitivities of commercial PVDF sensors of size $12 \mathrm{~mm}$ x $30 \mathrm{~mm}$ and $12 \mathrm{~mm} \times 10 \mathrm{~mm}$ were $0.008 \mathrm{~V} / \mathrm{N}$ and $0.0143 \mathrm{~V} / \mathrm{N}$. It can be seen that the reduction of the sensor active area increases the sensor sensitivity. That applies to both home-made and commercial sensors. The sensitivity of commercial sensors is smaller than for the home-made sensors.

The effect of protective layers on the home made PVDF sensor sensitivity was evaluated. The comparison of the sensor sensitivity with no additional protection layer of the Kapton tape and with one additional protection layer are shown in Figure 5. It is observed that sensor sensitivity decreases as the protective layer increases.

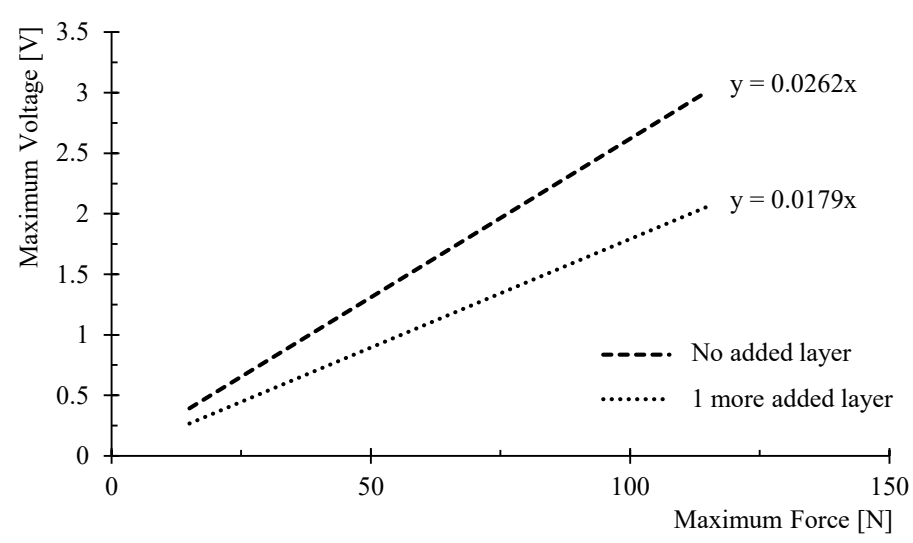

Fig. 5. The effect of one protection layer on the sensor sensitivity for home-made PVDF sensor. 


\section{Conclusion}

Home-made PVDF sensors were produced and tested and compared to commercial PVDF sensors. The photolithography method was successfully used for the fabrication of homemade PVDF sensors. The sensitivities of the home-made PVDF sensors were higher than for the commercial variants. This can be influenced by the composition of the protection lyres of the sensors. Note that for the measurement in the real cavitating flow high sensor protection is required which on the contrary decreases the sensor sensitivity. The optimum between the sensor sensitivity and the sensor protection should be further investigated. The sensitivity of the sensors is also influenced by the sensors active area. The smaller active area corresponds to the higher sensitivity of the sensor. The smaller active area also corresponds to the better special resolution of the impacts measurement.

The duration of the signal generated by the calibration method should be close to the signal generated during the impact in the real cavitating flow. The breaking pencil lead method [9] will be used to generate the calibration signal to prove this condition. The further work will be also focused on the application of the home-made PVDF films for the measurement of the cavitation impacts in the real cavitating flow.

Acknowledgment: This work was supported by the Student Grant Competition of the Technical University of Liberec under the project No. SGS-2020-5028 „Investigation of cavitation collapse by PVDF sensors“.

\section{References}

1. K. H. Kim, G. Chahine, J.-P. Franc, A. Karimi, Advanced experimental and numerical techniques for cavitation erosion prediction (Springer, Dordrecht, 2014)

2. Franc, J.-P. \& Michel, J.-M. (2004) Fundamentals of cavitation. Dordrecht, Kluwer.

3. J.-P. Franc, M. Riondet, A. Karimi, G. L. Chahine, J. Fluids Eng. 133, 12 (2011)

4. T. Momma, A. Lichtarowicz, Wear 186-187, 425-436 (1995)

5. H. Soyama, Y. Sekine, K. Saito, Measurement 44 (2011)

6. Y.-Ch. Wang, Y.-W. Chen, Exp. Therm. Fluid Sci. 32, 2 (2007)

7. A. S. Grinspan, R. Gnanamoorthy, Colloids Surf. A: Physicochem. Eng. Asp. 356, 162168 (2010)

8. J. Hujer, J.-B. Carrat, M. Müller, M. Riondet, J. Phys. Conf. Ser. 656 (1), 012051 (2015)

9. H. Soyama, A. Lichtarowicz, T. Momma, E. J. Williams, J. Fluids Eng. 120 (4), $712-$ 718 (1998) 